\title{
Efficacy of a mindfulness-based intervention programme with and without virtual reality support to reduce stress in university students: protocol for a randomized controlled pragmatic trial
}

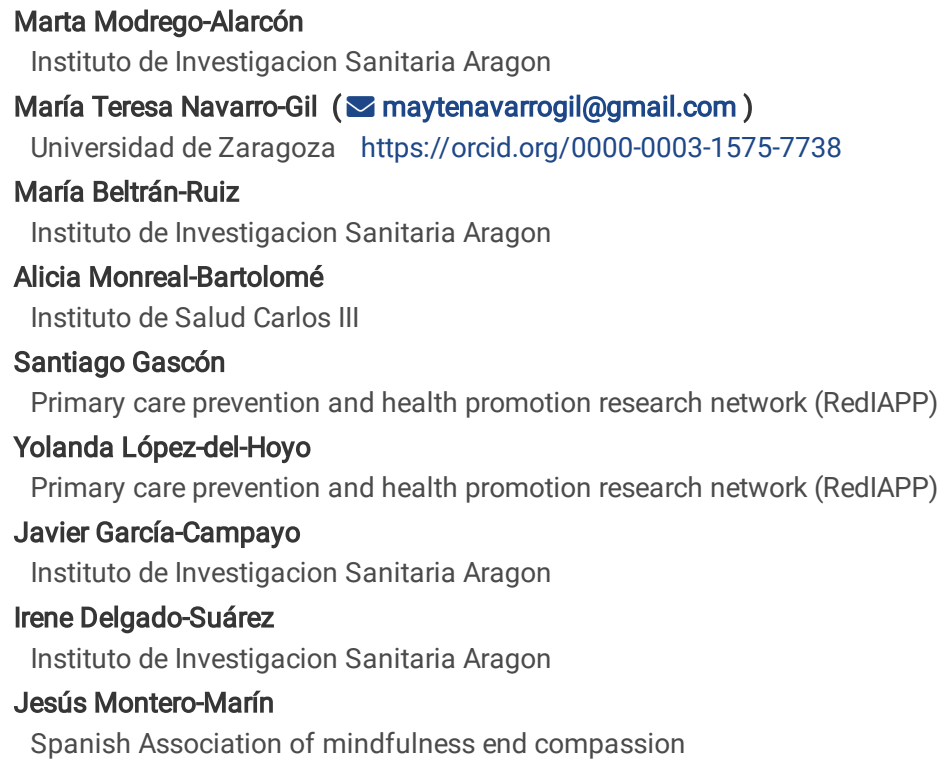




\section{Abstract}

Background: There is growing concern about mental health problems in university students. For this reason, mindfulness training is becoming increasingly popular in university settings. However, mindfulness-based interventions (MBIs) usually present high attrition rates. This trial aims to evaluate the efficacy of a MBI to reduce perceived stress and to improve the psychological well-being of university students, as well as to explore the capacity of virtual reality (VR) to enhance adherence to the intervention.

Methods: This study protocol presents a randomized controlled trial (RCT) involving assessment time points of baseline, posttreatment and 6-month followup. A total of 280 students from the Spanish National Distance Education University (UNED) or the University of Zaragoza will be randomly assigned to a mindfulness condition, a mindfulness condition complemented by VR, and a relaxation condition to serve as a control group. Stress will be the main outcome and will be measured using the 10-item self-report Perceived Stress Scale (PSS). Other well-being and academic functioning outcomes will be assessed, as well as variables that explore the impact of VR. Multilevel mixed-effects models will be calculated to estimate the efficacy of the programme, and effect size estimations will be carried out. Effects of VR in adherence to programme will be evaluated.

Discussion: Some strengths of this study are the RCT design, which includes a suitable active control group and a 6-month follow-up assessment; a large sample size of university students at different stages of their courses and a variety of degrees; and the incorporation of the VR support to facilitate completion of the MBI programme. Potential limitations are the voluntary participation of the students and the utilization of self-report measures.

Trial registration number: ClinicalTrials.gov NCT03771300.

\section{Background}

The mental health of university students is a noteworthy concern nowadays. Nineteen institutions of higher education across eight countries (Australia, Belgium, Germany, Mexico, Northern Ireland, South Africa, Spain, United States) participated in a project with the aim of estimating prevalence of common mental disorders among first-year college students, and found that $35 \%$ of the 13,948 respondents screened positive for at least one of the common lifetime disorders assessed and $31 \%$ screened positive for at least one 12-month disorder (1). Other similar studies indicate a high prevalence of psychological distress, anxiety and depression among university students (2-4) and even a high prevalence of suicidal thoughts and autolytic behaviours (5). Universities are thus challenged to set up effective preventive interventions in order to help students cope better with university life and improve their mental health. In this regard, a meta-analysis confirmed that cognitive, behavioural and mindfulness-based interventions are effective in reducing symptoms of anxiety in university students (6).

Mindfulness-based interventions (MBIs) are defined as a type of intervention whose main aim is to train the mind to adopt a non-judgemental present-focused awareness (7). In recent years, these interventions have been expanded and have proven to be effective for a variety of clinical and non-clinical populations (810). This expansion has allowed the benefits of mindfulness practice to be studied in university students (11-15). For instance, a recent study (16) with a large sample of 616 students from the University of Cambridge (UK) showed that the Mindfulness Skills for Students (MSS) programme reduced self-reported psychological distress in university students during the examination period compared to a group of support as usual, showing moderate effects.

The present study pursues the testing of this evidence in the Spanish context, while exploring the potential benefits of virtual reality (VR) when combined with the provision of mindfulness training to university students. The effectiveness of VR has been shown for the treatment of several psychological disorders, particularly anxiety disorders (17). Furthermore, current research has found promising results when it is incorporated into 'third wave psychotherapies' such as MBIs, although there is still only a relatively limited number of studies available (18). A recent study found a high acceptability of VR for the practice of mindfulness exercises, together with a significant increase in mindfulness state and an improved emotional state after only one VR mindfulness session in experienced meditators (19). It has recently been found that VR might enhance adherence to mindfulness programmes in patients suffering from generalized anxiety disorder (20). In general, MBIs suffer from important attrition rates $(10,21)$ and failure to consolidate practice habits might undermine possible benefits (22). The implementation of additional VR scenarios into the practice of mindfulness exercises could be well-accepted and useful among university students because of their high affinity with new technologies (23).

\section{Objectives}

In this context, the main objective of this study is to evaluate the efficacy of an MBI program to reduce stress in university students, in comparison with an active control group undertaking relaxation therapy, which has shown some evidence to reduce stress in this population (24-27). Other exploratory aims are as follows: (1) to analyse the efficacy of an MBI program for the improvement of psychological well-being and academic functioning, as well as dispositional mindfulness and self-compassion, in university students, in comparison with the active control group treated with relaxation exercises; (2) to evaluate whether there are differences in the adherence to practice in the mindfulness programme, according to the modality in which this mindfulness intervention has been applied (i.e. with or without VR support); (3) to examine the possible differential effectiveness of each of the mindfulness subgroups (i.e. with or without support of the VR) in comparison with the active control group treated with relaxation therapy; (4) to assess the effects of different mindfulness-based VR environments on the emotional and mindfulness states of university students, as well as to evaluate the sensation of immersion in the landscapes created by each VR environment.

Our main hypothesis is that the provision of mindfulness training will reduce perceived stress in university students to a greater extent than with relaxation training.

\section{Trial design}


This is a three-arm, parallel randomized and controlled, pragmatic superiority trial, involving three assessment time points (baseline, posttreatment and 6month follow-up). University student participants will be randomly assigned to three different intervention groups: mindfulness, mindfulness + VR, and relaxation therapy. The overall design and flow chart of the study can be seen in Figure 1. This protocol was designed in accordance with the SPIRIT 2013 statement (28). Trial registration number: ClinicalTrials.gov NCT03771300.

\section{Methods}

\section{Setting, eligibility criteria and recruitment}

Participants will be recruited from two Spanish universities in the province of Zaragoza (Spain). The participants will be students of the University of Zaragoza or the National Distance Education University (UNED), who will be recruited according to the following criteria: (a) $\geq 18$ years of age; (b) enrolled in undergraduate or master's degree courses at these universities; (c) enrolled in the faculties of social sciences or health sciences at the Zaragoza campus (in the case of University of Zaragoza students) or at the Calatayud campus (in the case of UNED students); (d) speaking and writing Spanish language; and (e) providing written informed consent before knowing the assignment group. No exclusion criteria will be considered to frame the work as a universal intervention for the promotion of health and stress management in university students.

Students will be recruited through the following three main procedures: (1) informative posters will be put up in university buildings containing a brief explanation of the study and the contact details; (2) several members of the university teaching staff will contact their students and send them an informative e-mail with a brief explanation of the study and the contact details; (3) different tuition centres, student organizations and support services for university students will also send their users an informative e-mail with a brief explanation of the study and the corresponding contact details.

\section{Sample size}

The sample size has been estimated taking as a reference a moderate difference $(d=0.40)$ between the total MBI group and the active control group in the main variable of perceived stress (Perceived Stress Questionnaire - PSS), as it has been observed in previous similar studies (29), accepting an alpha of 0.05 and a beta risk of 0.20 in a bilateral contrast, with a 2:1 allocation rate (so that two participants will be assigned to the mindfulness total group - one in the condition of VR support and another in the condition that does not have such support - for each control subject). This unequal randomization ratio design was established to gain additional exploratory information on the use of VR when added to the mindfulness treatment proposed (30, 31). Therefore, and under these circumstances, 225 participants will be required: 75 participants in the active control group of relaxation therapy, and 150 participants in the total MBI group. Taking into account all the participants, and assuming an attrition rate of around $25 \%$ (21), the total sample has been established at 280 university students.

\section{Randomization, allocation and masking of study groups}

The assignation of the subjects will be carried out after the baseline evaluation by a member of the research group, who has no knowledge about the study aims, through a simple random sequence generated by computer. Participants will be randomized into three groups ('mindfulness', 'mindfulness + VR', and 'relaxation'), considering the secondary objective of evaluating the effectiveness of each mindfulness subgroup separately. Nevertheless, taking into account the primary aim of isolating the common effects of both mindfulness subgroups, 'mindfulness' and 'mindfulness + VR' will work as an only one group of treatment. Posttreatment and 6-month follow-up measurements will be recorded by a different and independent assessor who will be blind to the study condition of participants. However, due to the characteristics of the intervention, providers and participants will be able to know what kind of intervention they are offering and receiving respectively, so this will be a single-blind study although all the participants will be treated through suitable active conditions. The interventions will be conducted in two cohorts: cohort 1 will be started in November 2018 and cohort 2 in April 2019 (Figure 2).

\section{Interventions}

Within the three conditions described below, motivational techniques will be used to increase adherence of participants to sessions and daily tasks. The participants will not receive any amount of economic compensation. In order to improve the adherence of participants to sessions, they will be sent reminders via WhatsApp of the start time of the session, a few hours prior to each session. Daily tasks aids, such as mindfulness or relaxation exercise audio recordings (depending on the condition) will also be sent through a weekly WhatsApp message in each group. All participants will be encouraged to do a daily task at home and will be asked to keep a daily written record of the number and duration of practice sessions.

-Mindfulness condition: In this group, $\mathrm{n}=93$ students will be participating in a programme structured around two central elements, mindfulness and compassion. The programme consists of 90-minute group sessions, held once a week over a space of 6 weeks, and is offered as an extra-curricular activity. Each sub-group of mindfulness condition will be formed by 15 or 16 participants, so there will be six sub-groups of this condition. The sessions combine components of theory and practice, using a methodology which prioritizes inquiry, reflection and debate among the group of students. The theory component will comprise mindfulness and compassion concepts set out over the length of the programme, in addition to others related to time management, stress, anxiety and the balance between personal life and academic/work life. The practical component of the program will consist of formal and informal mindfulness and self-compassion exercises under the guidance of a psychologist, who will be specifically trained in the application of the theoretical and practical components of the mindfulness programme to university students. Table 1 provides a summary of the structure and contents of the mindfulness programme by session.

-Mindfulness condition complemented by VR environments. This condition ( $n=93)$ is equivalent to the previous one (group sessions formed by 15 or 16 participants, held once a week over a space of 6 weeks and offered as an extra-curricular activity), except the duration of each session, which is reduced from 90 to 75 minutes. Consequently, the content of each mindfulness session is slightly shortened, but this time difference is replaced by an individual VR session 
held before or after the group mindfulness session. This short VR session is designed on an individual basis and consists of a mindfulness or selfcompassion exercise through VR. The VR kit comprises a set of Samsung GEAR VR goggles, a Samsung Galaxy S6 phone and optional headphones (https://www.psious.com/). The content and objectives of each VR scenario are specified in Table 2. In this condition, the mindfulness instructor will remain the same but the implementation of VR will be carried out by another psychologist who is specifically trained in the application of VR scenarios. Prior to the use of VR, the psychologist will check the participants' health. In general terms, the use of VR is not recommended for pregnant women; people suffering from hypertension, ear infections, epilepsy or vertigo; patients who have recently undergone surgery; or people who suffer from cardiovascular disease, psychosis or serious mental illness. For this reason, the psychologist will ensure that none of these conditions are present. The participant will be seated on a chair in a quiet and secure place.

-Relaxation condition: This group ( $\mathrm{n}=94)$ is based on the adapted version proposed by Bernstein and Borkovec of progressive muscle relaxation therapy (32). This programme also consists of 90-minute group sessions formed by 15 or 16 participants, held once a week over a space of 6 weeks, and offered as an extra-curricular activity. The relaxation programme involves the training of 16 muscle groups during the initial sessions, 7 muscle groups during the intermediate sessions, 4 muscle groups in the later stage, and only relaxation through recall in the final session. This programme is also complemented with visualizations, as it was originally proposed by Jacobson (33). Table 3 provides a summary of the programme structure and contents. All participants in this condition will be instructed by a psychologist specifically trained in the application of progressive muscle relaxation therapy.

\section{Outcomes and data collection}

Several outcomes will be measured and compared between the mindfulness (with and without VR support) and control groups. We will collect data on sociodemographic variables and experience in the use of new technologies (baseline), as well as on the primary and secondary outcomes (baseline, posttreatment and six month follow-up). The study primary outcome will be perceived stress. Secondary outcomes will be psychological wellbeing (e.g., anxiety, affectivity and emotional regulation), academic functioning (e.g., academic engagement, academic burnout and burnout clinical subtypes) and trait mindfulness and self-compassion. Other secondary VR measurements will be emotional intensity, state of mindfulness and sense of presence. All the measurements will be collected using paper-and-pencil surveys. In the last session, participants of all the groups will be called for a meeting to complete the posttreatment questionnaires. Participants who did not attend this meeting will be sent a message via WhatsApp, in which they will be informed about two possible dates to complete the questionnaires. Six months later, a similar strategy to complete the follow-up-intervention questionnaires will be used (all the students will be called for a meeting via WhatsApp). Participants who did not attend this meeting will be sent a new message with two possible dates. No outcome data will be collected for participants out of the referred meetings.

\section{Socio-demographic, use of new technologies and acceptability}

Demographic Information will be gathered by means of a questionnaire developed by our team, including: 1) date of birth; 2) sex; 3) marital status; 4) educational level; 5) employment status; 6) type of employment contract; 7) previous training in: a) relaxation, b) mindfulness and/or compassion, and c) VR (with two answer options for each one: yes, no); and 8) degree course currently being taken.

Experience on the use of new technologies will be measured with a brief version of the Independent Television Company SOP Inventory (ITC-SOPI) (34, 35). Preliminary analyses have demonstrated adequate psychometrics for the ITC-SOPI (34).

Acceptability of the intervention will be measured using an adapted version of the Credibility/Expectancy Questionnaire (36, 37). Participants will complete this scale before and after the intervention. The adapted questionnaire comprises 6 items rated from 0 ('not at all') to 10 ('very much'), with appropriate psychometric properties $(34,35)$.

\section{-Primary Outcome}

Perceived stress, measured by the 10 -item self-report PSS $(38,39)$, will be the primary outcome. Through the PSS, participants will be asked to rate how unpredictable, uncontrollable and overloaded they have found their life over the past month on a 5-point Likert-type scale (from $0=$ 'never' to $4=$ 'very often'). Higher scores indicate higher levels of stress. The Spanish PSS (40) provides a reliable and valid measure of perceived stress, with adequate psychometric properties ( $a=0.82$, test-retest, $r=0.77)$.

\section{-Secondary Outcomes}

Anxiety will be measured by the State-Trait Anxiety Inventory (STAI) (41-43). This inventory is a widely used, validated measure of anxiety and consists of 20 statements that evaluate how the participants feel at the present moment (state anxiety), and 20 statements that evaluate how the participants feel in general (trait anxiety). It uses a 0-3 Likert-type rating scale - the higher scores indicating greater anxiety levels - with good psychometric properties $(a=0.93)$ in university students (44).

Positive and negative affect will be measured by the Positive and Negative Affect Schedule (PANAS) (45). This self-report questionnaire consists of two 10item scales to measure both positive and negative affect. Each item is rated on a 5-point Likert-type scale from 1 ('not at all') to 5 ('very much'). Scores for each subscale are totalled, with higher scores indicating more positive or negative affect. The internal consistencies of the Spanish version of the positive and negative PANAS scales are 0.87 and 0.91 , respectively (46).

Emotional regulation will be measured by the Emotional Regulation Questionnaire (ERQ) (47). This 10-item scale has been designed to measure respondents' tendency to regulate their emotions in two ways: cognitive reappraisal (6 items) and expressive suppression (4 items). Participants respond using a 7-point Likert scale (from 1 = 'strongly disagree', to $7=$ 'strongly agree') where higher scores indicate increased use of regulatory strategies. The Spanish version of the 
ERQ shows adequate internal consistency, test-retest reliability, and convergent and discriminant validity, with reliability values of $a=0.79$ for reappraisal and $a=0.75$ for suppression (48).

Mindfulness will be measured by the Five Facet Mindfulness Questionnaire (FFMQ) (49). This 39-item self-report consists of five facets: observing (8 items), describing (8 items), acting with awareness (8 items), non-judging of inner experience (8-items) and non-reactivity to inner experience (7 items). Respondents indicate on a 5-point Likert-type scale the degree to which each item is generally true for them, ranging from 1 ('never or very rarely true') to 5 ('very often or always true'). Higher scores indicate higher levels of mindfulness. The facet scores can be combined to produce a total mindfulness score. The Spanish version of this scale (50) shows good internal consistency (Cronbach's alpha between 0.80 and 0.91 ).

Self-compassion will be measured by the Self-Compassion Scale (SCS) (51) Spanish version (52). This 26-item questionnaire assesses the components of self-compassion across three facets: self-kindness, common humanity and mindfulness. Higher scores indicate greater levels of self-compassion. The facet scores can be combined to produce a total self-compassion score (53). The ScS uses a 5-point Likert scale, ranging from 1 ('almost never') to 5 ('almost always'). The Spanish version has demonstrated to be a valid and reliable instrument $(a=0.87$; test-retest $=0.92)$.

Academic engagement will be measured by the Utrecht Work Engagement Survey Scale-Students (UWES-S) (54). This 17-item questionnaire includes three subscales: vigour (6 items), dedication (5 items) and absorption (6 items). The UWES is scored on a seven-point frequency Likert-type rating scale varying from 0 ('never') to 6 ('always'). Higher scores in each subscale indicate greater levels of engagement. The UWES Spanish version has demonstrated to have good psychometric properties (54).

Academic burnout will be measured by the Maslach Burnout Inventory Student Survey (MBI-SS) (54), where the references to work have been changed for references to study. This 15 -item questionnaire includes three subscales: exhaustion (5 items), cynicism (4 items) and efficacy (6 items). High scores in exhaustion and cynicism, and low scores in efficacy are indicative of burnout. Participants have to respond on a Likert-type scale with 7 options ranged from 0 ('never') to 6 ('always'). The psychometric properties of the MBI-SS Spanish validation have been observed to be adequate (54).

Burnout clinical subtypes will be measured by the 36-item Burnout Clinical Subtype Questionnaire (BCSQ-36) (55), which assesses the three following burnout subtypes: frenetic, under-challenged and worn-out. References to work will be changed to references to study. Each subscale comprises 4 items. Participants indicate the point to which they agree with each item using a Likert-type scale with 7 options ranging from 1 ('never') to 7 ('always'). Higher scores mean greater levels of burnout subtypes. The BCSQ-36 has demonstrated good psychometrics in the Spanish population (55).

\section{-Virtual Reality measurements}

A visual analogue scale (VAS) (56) will assess the intensity of different emotions before and after the VR intervention. A briefer version of the original measure (16 emotion items) will be used and it will be composed by 7 emotion items (happiness, sadness, anger, surprise, anxiety, relaxation/calm, vigour/energy). Participants can choose responses ranging from 1 ('not feeling the emotion at all') to 7 ('feeling the emotion extremely'). This 7-item scale has been successfully used in previous studies $(19,57)$.

State of mindfulness will be measured just before and after each VR session using an adaptation of the Mindful Attention Awareness Scale (MAAS-State) (5860). Item numbers $3,8,10,13$ and 14, has been designated to assess state mindfulness (58), and will be included. Each item will be rated on a 7-point Likerttype scale, between 0 ('not at all') and 6 ('very much'). Higher scores reflect greater levels of state mindfulness. This shorter 5 -item scale has been successfully used in previous studies (19).

Sense of presence in the VR scenarios will be measured just before and after each VR session, using 4 items with a 7-point Likert scale ranging from 1 to 7. These 4 items were adapted from the Slater, Usoh \& Steed Questionnaire (SUS) (61). The Spanish version has been used to measure sense of presence provided by VR, with $\mathrm{a}=0.92(19)$.

\section{Data monitoring and harms}

The Data Monitoring Committee (DMC) of this trial is a group composed of five experts: the person in charge of the project, the head of the group, an independent clinical psychologist, an independent psychiatrist and the psychologist responsible of data handling. The DMC will meet three times throughout the trial (just before starting, in the midst of the intervention and at the end of the trial), in order to evaluate the possible cases of participants presenting adverse effects and to ensure that the trial is carrying out correctly. The practice of relaxation may have associated paradoxical increases in tension, relaxation-induced anxiety and panic and decreases in sympathetic tone which can lead to nausea and vomiting (62). Adverse effects of meditation can include psychological effects such as emotional stress, confusion, disorientation and dependence on practice; psychopathological effects such as anxiety, deliriums and hallucinations; and/or physiological effects such as pain, sensorial dysfunction, exacerbation of neuromuscular/joint diseases, reduction in appetite and insomnia (63). In a recent multi-cultural study on the effects of meditation, $25.4 \%$ of the participants reported unwanted effects, but most of them were transitory with no need for medical assistance (64). On the other hand, VR is associated with uncomfortable physical responses such as nausea (burping, increased salivation, etc.), oculomotor symptoms (eyestrain, difficulty concentrating, etc.) and disorientation (dizziness, vertigo, etc.) (65-67). Any adverse effect which is referred from the participants during the sessions, along the trial or observed by the psychologists in charge of the groups will be communicated to the DMC. The DMC will have the responsibility of evaluating and dealing with such possible side effects, bringing it to the attention of the corresponding health professional, in case it is required. The DMC will recommend changing the group or terminating the programme if the unintended effects observed or reported are a consequence of the participation in the intervention and acquire clinical relevance from the point of view of the corresponding health professional. The DMC will function independently of the trial funder.

\section{Data analysis}


To report this randomized controlled trial (RCT) we will make use of the recommended CONSORT guidelines $(68,69)$. Paper-based data entry will be doublechecked revising possible out-of-range values. Socio-demographic data will be described at baseline by means of frequencies (percentages), medians (interquartile range) and means (SD), depending on the distribution of each variable. The treatment conditions will be compared to evaluate the success of randomization by means of chi-square (or Fisher when necessary), Kruskal-Wallis and one-way ANOVA tests, respectively. This strategy will also be used to analyse possible differences between groups in terms of preference for treatment conditions and credibility as a result of the treatment received.

\section{-Main analysis}

The efficacy of the general mindfulness programme - integrating both groups with and without the presence of VR support - compared to the relaxation control condition will be analysed according to the main PSS variable at posttreatment, which will be taken as a continuous outcome. It will be developed by means of a repeated measures design and an intention to treat (ITT) basis, by using multilevel mixed-effects regression models including time as an independent variable and subjects and presence/absence of VR support as random effect variables. For this, the restricted maximum likelihood (REML) estimation will be used, which produces unbiased estimates in case of small or unbalanced sample sizes (70). Non-standardized slopes and $95 \%$ confidence intervals $(95 \% \mathrm{Cl})$ will be calculated by adjusting the time of the year in which the programme was undertaken (temporality), and those socio-demographic variables that show significant differences between groups at baseline. The 'group $\mathrm{x}$ time' interaction will be considered in order to study the specific trajectories of each group throughout the intervention, and to determine whether the possible differences between the general mindfulness group and the relaxation group remain consistent over time. Effect size, as the differences between groups at post-test and at 6-month follow-up, will be assessed using Cohen's $d$ statistic, calculated by the combined standard deviation in the pre-test, thus weighing the differences between the corresponding marginal means (71). Effect sizes are defined as small, when $d \leq 0.2$; medium, when $d=0.5$; and large, when $d \geq 0.8$ (72).

\section{-Secondary analyses}

The efficacy of the general mindfulness programme - integrating both groups with and without the presence of VR support - compared to the relaxation control group regarding to the psychological well-being, academic functioning, trait mindfulness and self-compassion outcomes, will be calculated following the same analytical strategy used for the main analyses. Moreover, the efficacy of each mindfulness subgroup (with or without VR support) vs the control group as regards the main and secondary variables will be estimated, although the mixed regression model in this case will only include subjects as a random effect variable. Per protocol analysis will be carried out, considering only those participants who attend at least $\geq 50 \%$ of the sessions (73, 74 ). Sensitivity analysis will be developed to assess the effects of missing data, which will be replaced by multiple imputations based on chained equations as long as there are $<40 \%$ missing data in the corresponding outcomes and whether missing values are distributed at random (MAR) to ensure validity (75). Additionally, possible differences in emotional state, immersion in the VR environment and state of mindfulness in the mindfulness + VR subgroup will be explored through each of the VR environments, using mixed models.

Baseline socio-demographic and psychological characteristics and the fact of belonging to subgroups (with or without VR support) will be evaluated dependent on compliance with the programme. Participants will be considered to have completed the programme when they have attended at least half or more of the sessions $(73,74)$. The characteristics of the subgroups will be compared by means of the corresponding chi-square (or Fisher) tests, and the Student's t-test for unpaired groups.

No interim analyses before ending the study will be developed, unless DMC decided to prematurely terminate the study. An alpha level of 0.05 will be set, using a two-tailed test. The probability values relative to the main analysis will be adjusted according to Benjamini-Hochberg's correction for multiple comparisons. The secondary analyses will be considered as exploratory and thus they will not be corrected (76).

\section{Discussion}

Mental health of university students is being threatened as a result of the excessive pressure they are suffering to reach excellence, and that is manifest through high levels of stress (77). Thus, universities are being encouraged to provide preventive programmes for their students in order to improve their stress and mental health levels (6).

Mindfulness programmes are becoming a promising intervention in this regard, although there are several limitations across its research. Some of these limitations are the use of quasi-experimental or single-group research designs, small sample sizes, lack of follow-up measurements, and reliance only on selfreport measures (78). The present study is the first RCT that will be developed in the Spanish context aiming to lower perceived stress in university students by using a MBI programme. It pretends to overcome some of the referred previous limitations, using a randomized controlled trial with a suitable active control group treated with relaxation exercises, a relatively large sample comprising of 280 university students in different years and degree courses, and a 6-month follow-up assessment. Nonetheless, participation of the students will be voluntary, and the only measures used will be self-report questionnaires.

To the best of our knowledge, this is also the first RCT to explore the possibilities of VR use as an adjunct to the mindfulness programme in university students. One of these possibilities could be the improvement of adherence rates to completion to the programme, as it has been observed in other different populations suffering from generalized anxiety (20). An important barrier MBI programmes entail is the requirement of certain amount of discipline in the practice of meditation exercises (22) and as a consequence they suffer from high rates of attrition (10). It has been clearly established that failure to consolidate practice may undermine benefits related to the MBI interventions $(30,79)$. The attraction of new technologies such as VR produces to young people might favour completion to the programme in university students. Significant results from this trial in stress and well-being outcomes could be beneficial for the health of this population and would encourage further research along this line of intervention.

\section{List Of Abbreviations}


RCT: randomized controlled trial; MBI: mindfulness-based intervention; VR: virtual reality; UNED: Spanish National Distance Education University; PSS:

Perceived Stress Scale; MSS: Mindfulness Skills for Students; ITC-SOPI: Independent Television Company SOP Inventory; STAI: State-Trait Anxiety Inventory; PANAS: Positive and Negative Affect Schedule; ERQ: Emotional Regulation Questionnaire; FFMQ: Five Facet Mindfulness Questionnaire; SCS: Self-

Compassion Scale; UWES-S: Utrecht Work Engagement Survey Scale-Students; MBI-SS: Maslach Burnout Inventory Student Survey; BCSQ-36: Burnout Clinical Subtype Questionnaire; VAS: visual analogue scale; MAAS: Mindful Attention Awareness Scale -State; SUS: Slater, Usoh \& Steed Questionnaire; ITT: intention to treat.

\section{Declarations}

\section{Ethic statements}

Written informed consent will be obtained from all individual participants included in the study before randomization. They will be provided with detailed information about the study and informed that they may leave the study at any time, but they will not receive any remuneration or special grades for participating in the study. This study has been approved by the Ethical Committee of Aragon, Spain (PI18/325). Any important modification of the protocol will be communicated to this committee. All procedures performed in this study involving human participants are in accordance with the ethical standards of the 1964 Helsinki Declaration or comparable ethical standards. The data will be treated anonymously and will only be used for the purposes of the study. The confidentiality of participants will be guaranteed before, during and after the trial and will be protected by the Organic Law on Protection of Personal Data (15/1999 of December 13, LOPD) and all relevant EU legislation on privacy and data protection. All study information will be confined in secure drawers with limited access. Electronic data files will be password-protected and secured via advanced encryption standard (AES-256). In cases of unexpected effects, the DMC will be responsible for ensuring anonymity. This trial will be performed in compliance with the study protocol and with good clinical practice guidelines with the main aim of protecting and preserving human rights (80). Publication authorship of the final trial report will be based on making substantial contributions according to publication scholarly work in medical journals (81).

\section{Consent for publication}

Not applicable

\section{Availability of data and material}

The study is currently in progress, in the stage of data recruitment. Consequently, it is not possible to share the data. Once available, the results of the trial will be presented at national and international conferences and in peer-review journal publications. Anonymized data will be available on request under reasonable conditions.

\section{Competing interests}

The authors declare that they have no competing interests.

\section{Funding}

This project is not submitted to any competitive institutional call.

\section{Authors' contributions}

MMA, JGC and JMM conceptualized and designed the study. JMM and MMA wrote the first draft. JMM planned the statistical analysis. The mindfulness sessions were designed and elaborated by MMA and MNG, and the relaxation sessions by MBR and AMB. SG and YLDH critically reviewed the manuscript for intellectual content. JGC, MNG and JMM supervised all steps. All authors have read and approved the final manuscript.

\section{Acknowledgements}

MMA acknowledges the Spanish Ministry of Education, Culture and Sport (MECD) for an FPU grant (FPU15/00598). All authors would like to thank the support received by the DGA group (B17-17R) and the Network for Prevention and Health Promotion in primary Care (REDIAPP) from the "Instituto de Salud Carlos III" of the Spanish Ministry of Economy and Competitiveness, co-financed with European Union ERDF funds (RD16/0007/0005). We would also like to thank the Spanish Virtual Reality company, PSIOUS, which is providing us with the VR kit (Samsung GEAR VR goggles, Samsung Galaxy S6 phones and optional headphones), as well as the access to the different mindfulness scenarios (for more details of this company, visit the following website: https://www.psious.com).

\section{References}

1. Auerbach RP, Mortier P, Bruffaerts R, Alonso J. The WHO World Mental Health Surveys International College Student Project: Prevalence and Distribution of Mental Disorders. J Abnorm Psychol. 2018; 127(7): 623-638.

2. Dyrbye LN, Thomas MR, Shanafelt TD. Systematic review of depression, anxiety, and other indicators of psychological distress among US and Canadian medical students. Acad Med. 2006; 81 (4):354-373.

3. Ibrahim AK, Kelly SJ, Adams CE, Glazebrook C. A systematic review of studies of depression prevalence in university students. J Psychiatr Res. 2013; 47(3): 391-400. 
4. Stallman HM. Psychological distress in university students: A comparison with general population data. Australian Psychologist. $2010 ; 45(4): 249-257$.

5. Mortier P, Cuijpers P, Kiekens G, Auerbach RP, Demyttenaere K, Green JG, et al. The prevalence of suicidal thoughts and behaviours among college students: a meta-analysis. Psychol Med. 2018; 48: 554-65.

6. Regehr C, Glancy D, Pitts A. Interventions to reduce stress in university students: A review and meta-analysis. J Affect Disord. 2013; 148(1), 1-11.

7. Conley CS, Shapiro JB, Kirsch AC, Durlak JA. A meta-analysis of indicated mental health prevention programs for at-risk higher education students. J Couns Psychol. 2017; 64(2), 121-140.

8. Demarzo MM, Montero-Marin J, Cuijpers P, Zabaleta-del-Olmo E., Mahtani KR, Vellinga A et al. The efficacy of mindfulness-based interventions in primary care: A meta-analytic review. Ann Fam Med. 2015; 13(6), 573-582.

9. Gotink RA, Chu P, Busschbach JJ, et al. Standardised mindfulness-based interventions in healthcare: an overview of systematic reviews and metaanalyses of RCTs. PLoS ONE. 2015; 10:e0124344.

10. Khoury B, Lecomte T, Fortin G, Masse M, Therien P, Bouchard V, et al. Mindfulness-based therapy: a comprehensive meta-analysis. Clin Psychol Rev. 2013; 33:763-71

11. De Vibe M, Solhaug I, Tyssen R, et al. Mindfulness training for stress management: a randomised controlled study of medical and psychology students. BMC Med Educ. 2013;13:107.

12. Dvorakova K, Kishida M, Li J, Elavsky S, Broderick PC, Agrusti MR et al. Promoting healthy transition to college through mindfulness training with first-year college students: Pilot randomized controlled trial. J Am Coll Health. 2017; 65(4), 259-267.

13. Kuhlmann SM, Huss M, Bürger A, Hammerle F. Coping with stress in medical students: results of a randomized controlled trial using a mindfulness-based stress prevention training (MediMind) in Germany. BMC Med Educ. 2016; 16(1): 316.

14. Van Dijk I, Lucassen PL, Akkermans RP, van Engelen BG, van Weel C, Speckens AE. Effects of mindfulness-based stress reduction on the mental health of clinical clerkship students: A cluster-randomized controlled trial. Acad Med. 2017; 92(7), 1012-1021.

15. Lynch S, Gander ML, Nahar A, Kohls N, Walach H. Mindfulness-Based Coping With University Life: A Randomized Wait-List Controlled Study. SAGE Open Med. 2018; 8(1): 2158244018758379.

16. Galante J, Dufour G, Benton A, et al. Protocol for the Mindful Student Study: a randomised controlled trial of the provision of a mindfulness intervention to support university students' well-being and resilience to stress. BMJ Open 2016; 6: e012300.

17. Powers MB, Emmelkamp P. Virtual reality exposure therapy for anxiety disorders: a meta-analysis. Journal of Anxiety Disorders. 2008; 22:561-569.

18. Modrego-Alarcón M, Moreno LB, Correa M, Morillo H., Maicas RM, Garcia-Campayo J. Utilidad de la realidad virtual en la enseñanza y práctica de mindfulness: una revisión. C. Med. Psicosom. 2016; 120: 48-60.

19. Navarro-Haro MV, López-del-Hoyo Y, Campos D, Linehan MM, Hoffman HG, García-Palacios A. et al. Meditation experts try Virtual Reality Mindfulness: A pilot study evaluation of the feasibility and acceptability of Virtual Reality to facilitate mindfulness practice in people attending a Mindfulness conference. PloS one. 2017; 12(11): e0187777.

20. Navarro-Haro MV, Modrego-Alarcón M, Hoffman HG, López-Montoyo A, Navarro-Gil, M., Montero-Marin J et al. Evaluation of a Mindfulness-Based Intervention with and without Virtual Reality Dialectical Behavior Therapy® Mindfulness Skills training for the Treatment of Generalized Anxiety Disorder in Primary Care: A Pilot Study. Front Psychol. 2019; 10: 55.

21. Nam S, Toneatto T. The Influence of Attrition in Evaluating the Efficacy and Effectiveness of Mindfulness-Based Interventions. Int J Ment Health Addict. 2016; 14: 969-981.

22. Grossman P, Niemann L, Schmidt S, Walach H. Mindfulness-based stress reduction and health benefits: a meta-analysis. J. Psychosom. 2004; Res. 57: 35-43.

23. Furlong J, Davies C. Young people, new technologies and learning at home: Taking context seriously. Oxf Rev Educ. 2012; 38(1): 45-62.

24. Charlesworth EA, Murphy S, Beutler LE. Stress management skill for nursing students. Journal of Clinical Psychology. 1981; 37(2): 284-290.

25. Deckro G, Ballinger K, Hoyt M, Wilcher M, Dusek J, Myers $P$ et al. The evaluation of a mind/body intervention to reduce pychological distress and perceived stress in college students. J Am Coll Health. 2002; 50 (6): 281-287

26. Dehghan-Nayeri N, Adib-Hajbaghery M. Effects of progressive relaxation on anxiety and quality of life in female students: a non-randomized controlled trial. Complement Ther Med. 2011; 19 (4): 194-200.

27. Stephens RL. Imagery: a treatment for nursing student anxiety. J Nurs Educ. 1992; 31(7): 314-320.

28. Chan A-W, Tetzlaff JM, Altman DG, et al. SPIRIT 2013 Statement: Defining Standard Protocol Items for Clinical Trials. Ann Intern Med $2013 ; 158: 200$.

29. McConville J, McAleer R, Hahne A. Mindfulness training for health profession students-the effect of mindfulness training on psychological well-being, learning and clinical performance of health professional students: a systematic review of randomized and non-randomized controlled trials. Explore (NY) 2017; 13: 26-45.

30. Dumville JC, Hahn S, Miles JN, Torgerson DJ. The use of unequal randomisation ratios in clinical trials: a review. Contemp Clin Trials. 2006; $27(1)$, 1-12.

31. Hey SP, Kimmelman J. The questionable use of unequal allocation in confirmatory trials. Neurology. 2014; 82(1): 77-9.

32. Bernstein DA, Borkovec TD. Progressive Relaxation Training: A Manual for the Helping Professions. Champaign, IL: Research Press; 1973.

33. Jacobson E. Progressive Relaxation. Chicago, IL: University of Chicago Press; 1938.

34. Lessiter J, Freeman J, Keogh E, Davidoff J. A Cross-Media Presence Questionnaire: The ITC-Sense of Presence Inventory. Presence Teleoperators Virtual Environ [Internet]. 2001 Jun; 10(3): 282-97. 
35. Baños RM., Botella C, Alcañiz M, Liaño V, Guerrero B, Rey B. Immersion and emotion: their impact on the sense of presence. CyberPsychology Beh. 2004; 7(6), 734-741.

36. Borkovec TD, Nau SD. Credibility of analogue therapy rationales. J Behav Ther Exp Psychiatry [Internet].1972 Dec; 3(4):257-260.

37. Baños RM, Botella C, Guillen V, García-Palacios A, Quero S, Bretón-López J et al. An adaptive display to treat stress-related disorders: EMMA's World. Br J Guid \& Counc (internet). 2009;37(3): 347-356.

38. Cohen S, Kamarck T, Mermelstein R. A global measure of perceived stress. J Health Soc Behav. 1983; 24:385-396.

39. Cohen S, Williamson G. Perceived stress in a probability sample of the U.S. In Spacapam S, Oskamp S (Eds). The social psychology of health: Claremont Symposium on Applied Social Psychology. Newbury Park CA: Sage; 1988.

40. Remor E. Psychometric Properties of a European Spanish Version of the Perceived Stress Scale (PSS). 2006; 9(1):86-93.

41. Spielberger CD, Gorsuch RL, Lushene RE. Manual for the State-Trait Anxiety Inventory. Palo Alto, CA: Stanford University Press; 1970.

42. Bermúdez J. Análisis funcional de la ansiedad. Rev de Psicol. Gral y Aplic. 1978a; 153: 617e634.

43. Bermúdez J. Ansiedad y rendimiento. Rev de Psicol. Gral y Aplic. 1978b; 151:183e207.

44. Fonseca-Pedrero E, Paino Piñeiro M, Sierra Baigrie S, Lemos Giráldez S, Muñiz Fernández J. Propiedades psicométricas "Cuestionario de Ansiedad Estado/Rasgo" (STAI) en universitarios [Psychometric properties "Anxiety State/Trait Questionnaire" in college students]. Psicol Conductual. 2012; 20(3):547-62.

45. Watson D, Clark LA, Tellegen A. Development and validation of brief measures of positive and negative affect: the PANAS scales. J Pers Soc Psychol. 1988; 54: $1063 \mathrm{e} 1070$.

46. Sandín B, Chorot P, Lostao L, Joiner TE, Santed MA, Valiente RM. Escalas PANAS de afecto positivo y negativo: validación factorial y convergencia transcultural. Psicothema. 1999; 11(1): 37-51.

47. Gross JJ, John OP. Individual differences in two emotion regulation processes: Implications for affect, relationships, and well-being. J Pers Soc Psychol. 2003; 85: 348-362.

48. Cabello R, Salguero JM, Fernández-Berrocal P, Gross JJ. A Spanish adaptation of the emotion regulation questionnaire. Eur J Psychol Assess. 2013; 29(4):234-240

49. Baer RA, Smith GT, Lykins E, Button D, Krietemeyer J., Sauer S et al. Construct validity of the five facet mindfulness questionnaire in meditating and nonmeditating samples. Assessment. 2008; 15(3): 329-342.

50. Cebolla A, García-Palacios A, Soler J, Guillen V, Baños R, Botella, C. Psychometric properties of the Spanish validation of the Five Facets of Mindfulness Questionnaire (FFMQ). Eur. J. Psychiat. 2012; 26(2): 118-126.

51. Neff KD. The development and validation of a scale to measure self-compassion. Self Identity. 2003; 2(3): 223-250.

52. Garcia-Campayo J, Navarro-Gil M, Andrés E, Montero-Marin J, López-Artal L, Demarzo MM. Validation of the Spanish versions of the long (26 items) and short (12 items) forms of the Self-Compassion Scale (SCS). Health Qual Life Outcomes. 2014; 12: 4.

53. Neff KD, Tóth-Király I, Yarnell LM, Arimitsu K, Castilho P, Ghorbani N et al. Examining the factor structure of the Self-Compassion Scale in 20 diverse samples: Support for use of a total score and six subscale scores. Psychol. Assess. 2018: doi: 10.1037/pas0000629

54. Schaufeli WB, Martinez IM, Pinto AM, Salanova M, Bakker AB. Burnout and engagement in university students: A cross-national study. J Cross Cult Psychol. 2002; 33(5): 464-481.

55. Montero-Marín J, García-Campayo J. A newer and broader definition of burnout: Validation of the" Burnout Clinical Subtype Questionnaire (BCSQ36)". BMC Public Health, 2010; 10(1): 302.

56. Gross JJ, Levenson RW. Emotion elicitation using films. Cogn Emot [Internet]. 1995 Jan; 9(1):87-108.

57. Riva G, Ph D, Mantovani F, Ph D, Capideville CS, Ph D, et al. Affective Interactions Using Virtual Reality: The Link between Presence and Emotions. 2007; 10(1):45-56.

58. Brown KW, Ryan RM. The Benefits of Being Present: Mindfulness and Its Role in Psychological Well-Being. J Pers Soc Psychol. 2003; 84(4):822-48.

59. Cebolla A, Luciano JV, DeMarzo MP, Navarro-Gil M, Campayo JG. Psychometric properties of the Spanish version of the Mindful Attention Awareness Scale (MAAS) in patients with fibromyalgia. Health Qual Life Outcomes. 2013; 11(1):6.

60. Soler J, Tejedor R, Feliu-Soler A, Pascual JC, Cebolla A, Soriano J, et al. Propiedades psicométricas de la versión española de la escala Mindful Attention Awareness Scale (MAAS). Actas Esp Psiquiatr. 2012; 40:18-25

61. Slater M, Usoh M, Steed A. Depth of presence in virtual environments. Presence: Teleoperators Virtual Environ. 1994; 3(2): $130-144$.

62. Lazarus AA, Mayne TJ. (1990). Relaxation: Some limitations, side effects, and proposed solutions. Psychotherapy: Theory, Research, Practice, Training. 1990; 27(2): 261.

63. Lustyk MKB, Chawla N, Nolan RS, Marlatt GA. Mindfulness meditation research: issues of participant screening, safety procedures, and researcher

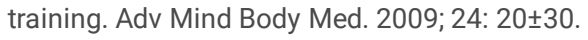

64. Cebolla A, Demarzo M, Martins P, Soler J, Garcia-Campayo J. Unwanted effects: Is there a negative side of meditation? A multicentre survey. PloS one. 2017; 12(9):e0183137.

65. LaViola JrJJ. A discussion of cybersickness in virtual environments. ACM SIGCHI Bulletin. 2000; 32(1): 47-56.

66. Kennedy RS, Lane NE, Berbaum KS, Lilienthal MG. Simulator sickness questionnaire: an enhanced method for quantifying simulator sickness. Int. J. Aviat. Psychol. 1993; 3(3): 203-220. 
67. Kim YY, Kim HJ, Kim, EN, Ko HD, Kim HT. Characteristic changes in the physiological components of cybersickness, Psychophysiology. 2005; 42(5): 616625.

68. Boutron I, Moher D, Altman DG, Schulz KF, Ravaud P. Extending the CONSORT statement to randomized trials of nonpharmacologic treatment: explanation and elaboration. Ann Intern Med. 2008;148(4), 295-309.

69. Moher D, Hopewell S, Schulz KF, Montori V, Gøtzsche PC, Devereaux PJ, et al. CONSORT. CONSORT 2010 explanation and elaboration: updated guidelines for reporting parallel group randomised trials. Int J Surg 2012; 10(1):28-55.

70. Egbewale BE, Lewis M, Sim J. Bias, precision and statistical power of analysis of covariance in the analysis of randomized trials with baseline imbalance: a simulation study. BMC Med Res Methodol. 2014; 14: 49.

71. Morris SB. Estimating effect sizes from pretest-posttest-control group designs. Organ Res Methods. 2008; 11:364-386.

72. Cohen J. Statistical power analysis for the behavioral sciences. $2^{\circ} \mathrm{Ed}$. Hillsdale, NJ: Erlbaum; 1988.

73. Kuyken W, Byford S, Taylor RS, Watkins E, Holden E, White K et al. Mindfulness-based cognitive therapy to prevent relapse in recurrent depression. J Consult Clin Psychol. 2008; 76: 966-978.

74. Gu J, Strauss C, Bond R, et al. How do mindfulness-based cognitive therapy and mindfulness-based stress reduction improve mental health and wellbeing? A systematic review and meta-analysis of mediation studies. Clin Psychol Rev. 2015; 37: 1-12.

75. White IR, Royston P, Wood AM. Multiple imputation using chained equations: Issues and guidance for practice. Stat Med. 2011; 30(4): $377-399$.

76. Feise RJ. Do multiple outcome measures require p-value adjustment? BMC Med Res. 2002; 2(1): 8.

77. Shields N. Stress, active coping and academic performance among persisting and non-persisting college students. J Appl Biobehav Res. 2001; 6(2): 6581.

78. Bamber MD, Kraenzle Schneider J. Mindfulness-based meditation to decrease stress and anxiety in college students: A narrative synthesis of the research. Educational Research Review. 2016; 18:1-32.

79. Crane C, Crane RS, Eames C, Fennell MJV, Silverton S, Williams JMG et al. The effects of amount of home meditation practice in mindfulness based cognitive therapy on hazard of relapse to depression in the staying well after depression trial. Behav. Res. Ther. 2014; 63: 17-24

80. Vijayananthan A, Nawawi 0. The importance of Good Clinical Practice guidelines and its role in clinical trials. Biomed Imaging Interv J. $2008 ; 4(1)$ :e5.

81. International Committee of Medical Journal Editors (ICMJE). (Internet) (2017 Nov 2). Available from: http://www.icmje.org

\section{Tables}

able 1. Structure and contents of the mindfulness programme

Note. This mindfulness programme has been designed by some of the authors of the present article.

\section{Virtual Reality Scenarios}

\section{ity Content and objective}

the This exercise is set in a conscious walking environment and consists of two component exercises. In the first one, the participant is instructed to focus attent exercise, the participant is instructed to be aware of bodily sensations while observing a lemon.

n The simulation of a human figure is used in this exercise. Throughout it, different body areas of this figure are highlighted (by means of blue bubbles) whil human figure is fixed and three-dimensionality is provided by the mountain landscape around the human figure.

s This exercise is set in a spring landscape. The participant will be asked to focus attention on several elements of the natural landscape while walking. When exercise, the camera will move away to ask the participant to observe the landscape from the new perspective.

$\mathrm{x}$ in

This exercise helps to find calm and serenity in a difficult situation such as an examination. The participant is immersed in an exam situation taking place

The first part of this exercise is based on identifying and recreating a life situation in which the participant has experienced a negative emotion. In the secor rate negative emotion.

This exercise is set in a desert scenario. It is the shortest exercise and is based on 1) identifying and being aware of three positive aspects of the participant

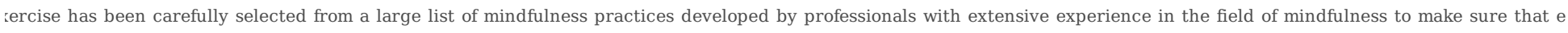
o the contents provided in each group session and supports the learning of the session.

Table 3. Structure and contents of the relaxation programme 


\begin{tabular}{ll}
\hline 1.-An introduction to mindfulness & -Group introductions and presentation of \\
& the objectives \\
& -Mindfulness and its usefulness in daily life \\
& -Attention and awareness. Automatic pilot \\
& -Information on posture \\
& -Breathing as an anchoring point
\end{tabular}

2.-What are thoughts and how can I relate to them?
-Thoughts and managing thoughts. Figure of the observer of thoughts -The world of interpretations and judgements. The beginner's mind, balancing the pros and contras. Selffulfilling prophecies

-Body sensations as an anchoring point
-Breathing exercise

-Simple exercises (listening to sounds, contact with the soles of the feet, contact with clothing)

-Raisin meditation exercise. Savouring

-De-mechanisation of a routine activity

-Hello, thanks and goodbye -Visual metaphors of the mind -Three compassionate minutes exercise

\section{3.-The body, much more than a 'vehicle for transporting the mind'/Time} difficulties and adjustments

-'The thing called the body'

-Body movement and body sensations as an anchoring point

-Time management. Procrastination.
-Body scan exercise

-Mindful walking
-Mindful body movements

-Mindful body movements
-Mindful walking

Mindful sighing

-Recording the time

\begin{abstract}
4.-What are emotions and how can I relate to them? The reality of suffering and acceptance
\end{abstract}

-Emotions and managing emotions. The observer. Embracing emotion

-Pain or primary suffering and secondary suffering. Acceptance

-Preparing ourselves for self-compassion kind attention

\section{5.-Challenges at university/Self- compassion: a new way of being} with yourself

\section{-Stress, anxiety and other challenges at} university

-Knowing and managing your own critical voice

-Self-compassion and its usefulness in daily life

$\begin{array}{ll}\text {-Compassionate breathing } & \text {-Inhaling/exhaling in difficult } \\ \text { exercise } & \text { situations } \\ \text {-Compassionate body scan } & \\ \text { exercise } & \end{array}$

exercise
-Identifying and substituting -Recording stressful events the destructive self-critical voice for one that is more constructive

-Compassionate coping in difficult situations -Receiving good wishes

\begin{tabular}{ll}
\hline 6.-Mindfulness and self- & -Recap of the main concepts \\
compassion, new allies in our day & -How to incorporate what has been learnt \\
to day & into daily life and into the university study \\
& context \\
& -Group farewell
\end{tabular}

\section{-Revision of the main formal and} informal mindfulness and compassion exercises -Calming contact exercise -A compassionate letter to yourself

\begin{tabular}{|c|c|c|}
\hline At the beginning of the session & Exercises & At the end of the session \\
\hline $\begin{array}{l}\text {-Group introductions and presentation of the } \\
\text { objectives } \\
\text {-Basic principles of progressive muscle } \\
\text { relaxation } \\
\text {-Systematic tension and release of } 16 \text { muscle } \\
\text { groups }\end{array}$ & $\begin{array}{l}\text {-Progressive muscle relaxation exercise with } 16 \\
\text { muscle groups } \\
\text {-Imagination training }\end{array}$ & $\begin{array}{l}\text {-Identification of sensations and difficulties with } \\
\text { relaxation }\end{array}$ \\
\hline $\begin{array}{l}\text {-Sharing experience of daily tasks } \\
\text {-Visualization techniques }\end{array}$ & $\begin{array}{l}\text {-Progressive muscle relaxation exercise with } 16 \\
\text { muscle groups } \\
\text {-Orange exercise(visualization) }\end{array}$ & $\begin{array}{l}\text {-Identification of sensations and difficulties with } \\
\text { relaxation }\end{array}$ \\
\hline $\begin{array}{l}\text {-Sharing experience of daily tasks } \\
\text {-Systematic tension and release of } 7 \text { muscle } \\
\text { groups }\end{array}$ & $\begin{array}{l}\text {-Progressive muscle relaxation exercise with } 7 \text { muscle } \\
\text { groups } \\
\text {-The beach exercise(visualization) }\end{array}$ & $\begin{array}{l}\text {-Identification of sensations and difficulties with } \\
\text { relaxation }\end{array}$ \\
\hline -Sharing experience of daily tasks & $\begin{array}{l}\text {-Progressive muscle relaxation exercise with } 7 \text { muscle } \\
\text { groups } \\
\text {-Landscape exercise(visualization) }\end{array}$ & $\begin{array}{l}\text {-Identification of sensations and difficulties with } \\
\text { relaxation }\end{array}$ \\
\hline $\begin{array}{l}\text {-Sharing experience of daily tasks } \\
\text {-Systematic tension and release of } 7 \text { muscle } \\
\text { groups }\end{array}$ & $\begin{array}{l}\text {-Progressive muscle relaxation exercise with } 4 \text { muscle } \\
\text { groups } \\
\text {-The globe and white light exercise(visualization) }\end{array}$ & $\begin{array}{l}\text {-Identification of sensations and difficulties with } \\
\text { relaxation }\end{array}$ \\
\hline $\begin{array}{l}\text {-Sharing experience of daily tasks } \\
\text {-Relaxation through recall }\end{array}$ & $\begin{array}{l}\text {-Relaxation through recall } \\
\text {-Mental relaxation and 'the perfect day 'exercise } \\
\text { (visualization) }\end{array}$ & $\begin{array}{l}\text {-Identification of sensations and difficulties with } \\
\text { relaxation }\end{array}$ \\
\hline
\end{tabular}

Note. Adapted version proposed by Bernstein and Borkovec (1973) of the Progressive Muscle Relaxation.

\section{Figures}




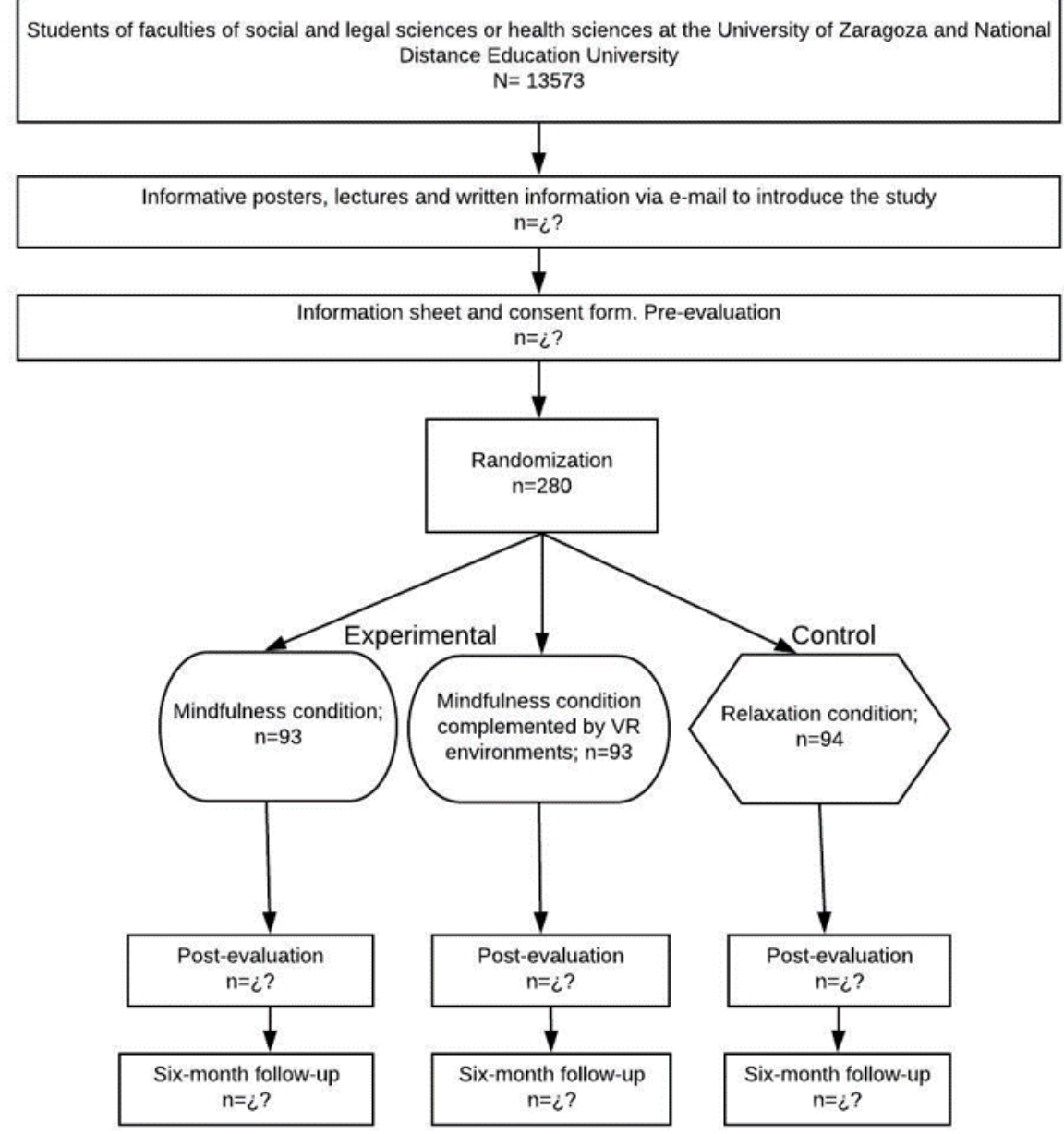

\section{Figure 1}

Flow chart of the study

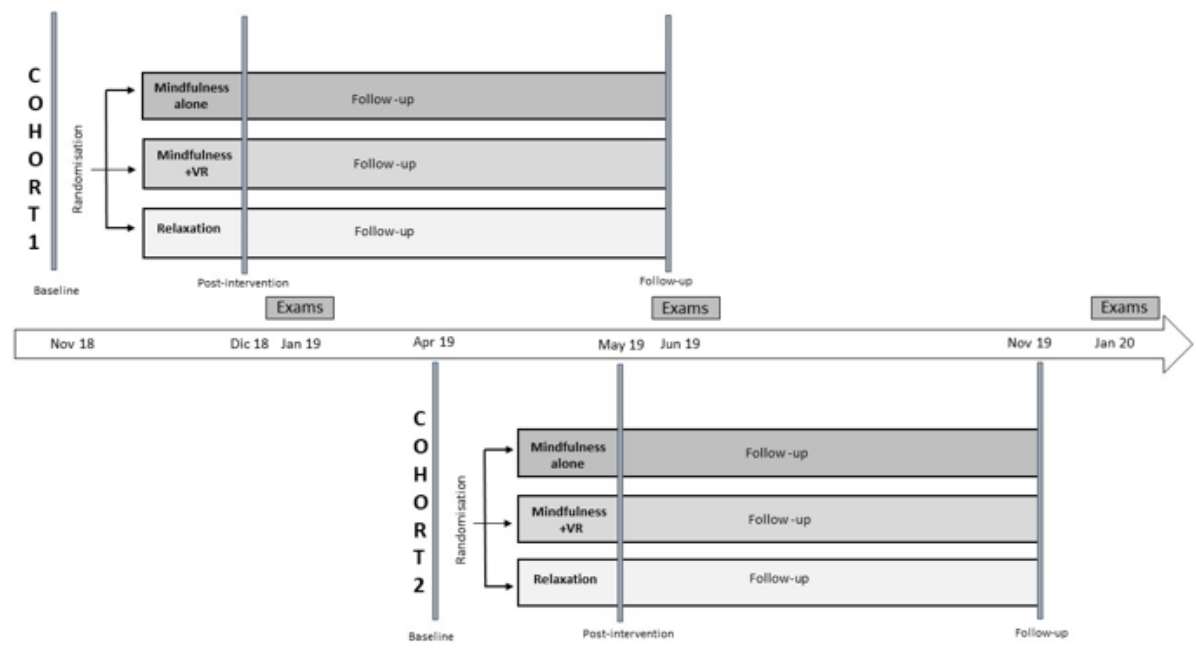

\section{Figure 2}

Timeline 

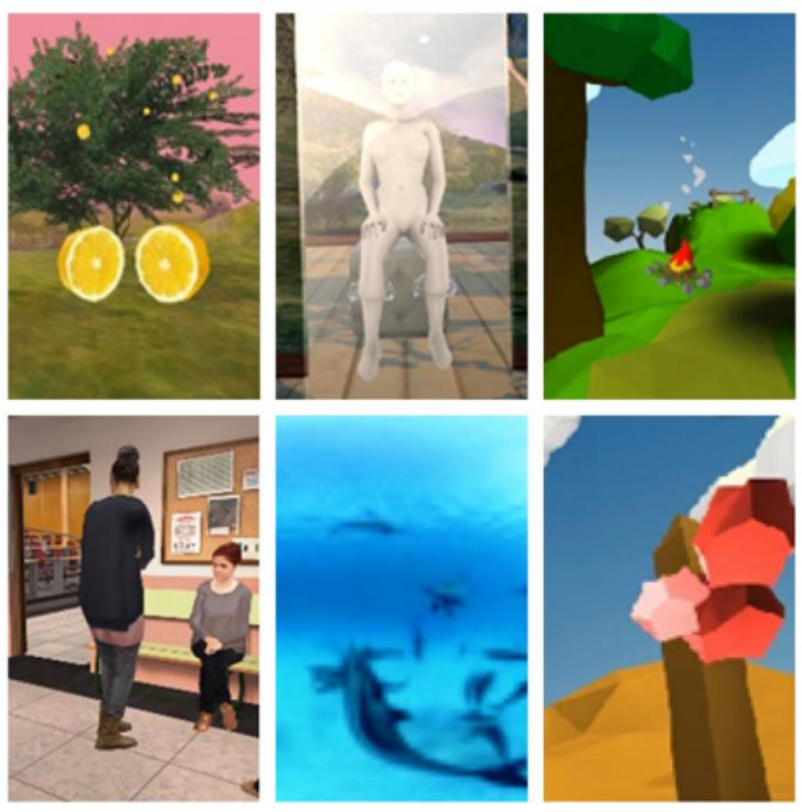

Figure 3

Virtual Reality Scenarios 